\title{
Commentary Barbiturates for the treatment of intracranial hypertension after traumatic brain injury
}

\author{
Sarice L Bassin and Thomas P Bleck
}

Department of Neurology, Northwestern University Feinberg School of Medicine, N Lake Shore Drive, Chicago, Illinois 60611, USA

Corresponding author: Sarice L Bassin, sbassin@nmff.org

Published: 20 October 2008

Critical Care 2008, 12:185 (doi:10.1186/cc7020)

This article is online at http://ccforum.com/content/12/5/185

(c) 2008 BioMed Central Ltd

See related research by Pérez-Bárcena et al., http://ccforum.com/content/12/4/R112

\begin{abstract}
In their article on the use of barbiturates for the treatment of intracranial hypertension after traumatic brain injury, Perez-Barcena and colleagues conclude that thiopental was more effective than pentobarbital in decreasing intracranial pressure. Here we discuss the limitations of this study and review areas of controversy surrounding barbiturate use in neurocritical care.
\end{abstract}

Raised intracranial pressure (ICP) after traumatic brain injury (TBI) is common and associated with increased risk for death and disability. Despite decades of animal and human research, successful prevention and treatment of this deadly complication largely eludes the medical community. Because of the considerable heterogeneity and severity of the disease, well designed, prospective, randomized studies in neurotrauma are rare. All academic attempts to generate reliable trial data are noteworthy.

In this context, we enthusiastically applaud Pérez-Bárcena and colleagues [1] for their thoughtful and ambitious research. The study evaluates the use of two barbiturates, pentobarbital and thiopental, in the treatment of refractory intracranial hypertension after TBI. Forty-four patients were randomly assigned to receive pentobarbital or thiopental after first-level measures had failed to control ICP. In the pentobarbital and thiopental groups, ICP was controlled in $18 \%$ and $50 \%$ of patients, respectively, without any statistically significant difference between groups in the rate of infectious complications or hemodynamic compromise. Glasgow Outcome Scale (GOS) scores at 6 months revealed a poor neurologic outcome (death, vegetative state, and severe disability) in 17 and 12 patients in the pentobarbital and thiopental groups, respectively. No statistical analysis of GOS scores was reported, but the study was not powered to detect a difference in outcome.
The study has several limitations, which the authors themselves note. Specifically, cranial computed tomography revealed bilateral brain swelling in significantly more patients in the pentobarbital group, and more patients in the thiopental group had evacuated lesions or no swelling at all. In addition, the doses of barbiturate were not equivalent between groups. To compensate for this, the authors attempted to ensure equivalent potency by titrating the dose to electrographic burst suppression (EBS) or flat pattern. However, the number of patients in each group that reached EBS was not reported; therefore, it is unclear whether bioequivalent doses were actually achieved between groups. Previous research has shown that the serum concentration at which EBS is reached varies between patients, and serum and cerebrospinal fluid levels correlate poorly with EBS [2]. Therefore, EBS is not necessarily an accurate surrogate for barbiturate dose. Given that thiopental is more lipophilic than pentobarbital and was infused at a higher initial maintenance rate, it is possible that the thiopental group maintained a higher cerebral concentration of barbiturate [3].

Despite these potential confounders, the relevance of this trial cannot be overstated. In the current age of multimodality monitoring, individualized treatment paradigms, and combination therapy, these data have important implications and bring to the forefront a number of questions.

What is the appropriate goal of barbiturate therapy - ICP control, EBS, neuroprotection, or a combination of these? Experimental models of ischemia demonstrate that anesthetic doses of barbiturates provide no additional attenuation of brain free fatty acid release than subanesthetic doses [4]. In models of focal infarction, animals that were anesthetized with pentobarbital dosed to preserve an active electroencephalogram had equivalent reductions in infarct volume 
as those anesthetized to EBS [5]. Barbiturates lessen the release of $\mathrm{S}-100 \mathrm{~B}$, excitatory neurotoxins, and amino acid markers of energy failure, but it has not been proven that EBS is a requirement for these neurochemical changes $[6,7]$. These data cast doubt on the argument that patients benefit maximally from barbiturate protocols that include EBS as a therapeutic target, especially given the frequent adverse effects of hypotension and immune dysfunction.

Nevertheless, several studies convincingly demonstrate that barbiturates can treat elevated ICP, particularly in patients who are refractory to other management strategies $[8,9]$. If barbiturates can lower ICP and provide neuroprotection even without EBS, then why has an association with good outcomes not been realized in clinical trials [10]? Perhaps by the time barbiturates are employed for refractory ICP - often several days after the trauma - the opportunity for the drugs to prevent secondary injury has passed. Alternatively, it is plausible that only specific pathophysiologic and cerebral hemodynamic profiles will respond to barbiturate treatment. Pérez-Bárcena and coworkers [1] reported that the association of focal lesions with ICP control was 3.6 times higher than that for diffuse lesions. Improved pressure reactivity indices and cerebral tissue oxygen tension in response to barbiturates, even in the presence of continued elevations in ICP, predict a favorable outcome [8]. These findings raise the possibility that in select patients barbiturates have the ability to prevent localized ischemia, reverse dysautoregulation, and improve cerebral oxygenation. Finally, barbiturates may indeed provide an outcome benefit, but were not superior to other drugs - including opiates, mannitol, hypertonic saline, and benzodiazepines - that were used in controls.

Despite insufficient evidence that either pentobarbital or thiopental improves outcomes after TBI, or that one drug is better than the other, the future for barbiturates still looks bright. Early combination therapy of barbiturates with hypothermia or progesterone, in concert with multimodality invasive neuromonitoring, holds promise in the treatment of this devastating condition [11-14].

\section{Competing interests}

The authors declare that they have no competing interests.

\section{References}

1. Pérez-Bárcena J, Llompart-Pou JA, Homar J, Abadal JM, Raurich $\mathrm{JM}$, Frontera G, Brell M, Ibanez J, Ibanez J: Pentobarbital versus thiopental in the treatment of refractory intracranial hypertension in patients with traumatic brain injury: a randomized controlled trial. Crit Care 2009, 12:R112.

2. Winer JW, Rosenwasser RH, Jimenez F: Electroencephalographic activity and serum and cerebrospinal fluid pentobarbital levels in determining the therapeutic end point during barbiturate coma. Neurosurgery 1991, 29:739-741.

3. Timbrell J: Principles of Biochemical Toxicology. Philadelphia, PA: Taylor \& Francis; 2000.

4. Shiu GK, Nemoto EM, Nemmer J: Dose of thiopental, pentobarbital, and phenytoin for maximal therapeutic effects in cerebral ischemic anoxia. Crit Care Med 1983, 11:452-459.
5. Warner DS, Takaoka S, Wu B, Ludwig PS, Pearlstein RD, Brinkhous $A D$, Dexter F: Electroencephalographic burst suppression is not required to elicit maximal neuroprotection from pentobarbital in a rat model of focal cerebral ischemia. Anesthesiology 1996, 84:1475-1484.

6. Korfias S, Stranjalis G, Boviatsis E, Psachoulia C, Jullien G Gregson B, Mendelow AD, Sakas DE: Serum S-100B protein monitoring in patients with severe traumatic brain injury. Intensive Care Med 2007, 33:255-260.

7. Persson L, Hillered L: Chemical monitoring of neurosurgical intensive care patients using intracerebral microdialysis. $J$ Neurosurg 1992, 76:72-80.

8. Thorat JD, Wang EC, Lee KK, Seow WT, Ng I: Barbiturate therapy for patients with refractory intracranial hypertension following severe traumatic brain injury: its effects on tissue oxygenation, brain temperature and autoregulation. J Clin Neuroscience 2008, 15:143-148.

9. Eisenberg HM, Frankowski RF, Contant CF, Marshall LF, Walker MD: High-dose barbiturate control of elevated intracranial pressure in patients with severe head injury. J Neurosurg 1988, 69:15-23

10. Roberts I. Barbiturates for acute traumatic brain injury. Cochrane Database Syst Rev 2000, 2:CD000033.

11. Varathan Sriranganathan, Shibuta S, Shimizu T, Varathan V, Mashimo T: Hypothermia and thiopentone sodium: individual and combined neuroprotective effects on cortical cultures exposed to prolonged hypoxic episodes. I Neurosci Res 2002, 68:352-362.

12. Muroi C, Frei K, El Beltagy M, Cesnulis E, Yonekawa Y, Keller E: Combined therapeutic hypothermia and barbiturate coma reduces interleukin- 6 in the cerebrospinal fluid after aneurysmal subarachnoid hemorrhage. J Neurosurg Anesthesio/ 2008, 20:193-198

13. Zausinger S, Westermaier T, Plesnila N, Steier HJ, SchmidElsaesser R: Neuroprotection in transient focal cerebral ischemia by combination drug therapy and mild hypothermia. Stroke 2003, 34:1526-1532.

14. Majewska MD, Schwartz RD: Pregnenolone-sulfate: an endogenous antagonist of the gamma-aminobuyric acid receptor complex in brain? Brain Res 1987, 404:355-360. 